Cahiers de recherches médiévales

\title{
Sur la vergogna dans le Décaméron
}

Entre pudeur et verecundia

Philippe Guérin

\section{(2) OpenEdition \\ Journals}

Édition électronique

URL : https://journals.openedition.org/crm/11535

DOI : 10.4000/crm. 11535

ISSN : 1955-2424

Éditeur

Honoré Champion

Édition imprimée

Date de publication : 15 juin 2009

Pagination : 295-311

ISSN : $1272-9752$

Référence électronique

Philippe Guérin, «Sur la vergogna dans le Décaméron », Cahiers de recherches médiévales [En ligne], 17| 2009, mis en ligne le 15 juin 2012, consulté le 15 décembre 2022. URL : http://

journals.openedition.org/crm/11535; DOI : https://doi.org/10.4000/crm.11535 


\title{
RM
}

\section{Sur la vergogna dans le Décaméron : entre pudeur et verecundia}

\begin{abstract}
After describing and illustrating by reference to Dante's Convivio the main lines of the context in which the question of passions in Boccaccio's Decameron can be understood, the study focuses on one passion in particular : "vergogna". "Vergogna" is in fact composed of affects that cannot be reduced to one another. The study analyses one of them that appears to play a regulatory function, not only in the mimesis of the "human comedy" as respresented by the different narrators, but also metatextually, in terms of the strategy the author deploys. This strategy not only provides the text - taken as a unitarian whole - with an efficient principle of internal cohesion ; it also calls into play quite specific options as regards "practical" philosophy.
\end{abstract}

Résumé : Après avoir tracé dans ses grandes lignes le cadre général-illustré notamment par le Convivio de Dante - où s'inscrit la question des passions dans le Décaméron de Boccace, l'étude s'attache à préciser les enjeux d'une passion particulière, la "vergogna». Elle montre que celle-ci se décompose en réalité en affects non exactement réductibles l'un à l'autre. Elle se conclut par l'examen de l'un d'eux, qui a une fonction régulatrice, non seulement dans la mimèsis de la "comédie humaine " telle que la représentent les différents narrateurs, mais, sur le plan métatextuel, dans la stratégie d'auteur mise en æuvre, celle-là même qui non seulement fournit au texte pris dans sa globalité de recueil unitaire un principe efficace de cohésion interne, mais met en jeu des options bien précises en matière de philosophie "pratique».

L'auteur du Décaméron n'est pas un théoricien. Boccace a pour ambition principale de «descendre [...] jusqu'aux événements et aux actes des humains $»^{1}$, à travers les récits des narrateurs de l' «honnête» et «joyeuse compagnie $»^{2}$. Il n'est pas davantage un redresseur de torts, armé d'une morale préétablie et contraignante ${ }^{3}$. Notre œil mortel ne saurait pénétrer les arcanes de l'esprit divin et nous ne pouvons nous appuyer que sur ce qui nous tombe sous les yeux et, par notre «jugement»,

\footnotetext{
${ }^{1}$ Dans le texte original : «il discendere [...] agli avvenimenti e agli atti degli uomini »; je cite d'après l'éd. suivante : Giovanni Boccaccio, Decameron, nuova edizione rivista e aggiornata, a cura di V. Branca, Torino, Einaudi, 1992 3 , I, p. 78 (nouvelle I3, 3). Pour les citations en français, v. la (très intéressante) traduction récente, Boccace, Le Décaméron, traduction nouvelle et postface de G. Clerico, préface de P. Laurens, dossier de C. et P. Laurens, Paris, Gallimard («Folio classique »), 2006.

2 « onesta brigata » (Proemio, 13), « lieta brigata » (I Introduzione, 103).

${ }^{3}$ Cf. par exemple le propos introductif de la narratrice en X 9, 4 («E se noi qui per dover correggere i difetti mondani o pur per riprendergli fossimo, io seguiterei con diffuso sermone le sue parole [i.e. le commentaire sur le prix de l'amitié par quoi la narratrice de la nouvelle précédente conclut son tour de parole] ; ma per ciò che altro è il nostro fine $[\ldots] »)$.
}

Cahiers de Recherches Médiévales, 17, 2009 
nous en faire une simple opinion. ${ }^{4}$. Introduite par les paliers successifs que constituent le récit-cadre, dont l'introduction à la première journée est la grandiose ouverture, et les deux premières nouvelles, c'est donc la phénoménologie concrète de la "comédie humaine ${ }^{5}$ qui, dans ses infinies variations, et doublée du plaisir sans mélange de la narration, sollicite au premier chef l'attention de Boccace. Si un enseignement d'ordre général se dégage tout de même avec force des cent nouvelles $^{6}$, il s'agit d'une morale toute provisoire, éminemment pragmatique. On pourrait l'énoncer comme suit: comment, pour les personnages qui peuplent ces nouvelles, trouver, inventer de fois en fois, les solutions les meilleures pour arriver à leurs fins, en tirant parti des situations, sinon totalement inédites, jamais totalement prédictibles, auxquelles ils se trouvent confrontés ; ou pour se tirer des mauvais pas, dont, à côté de la «fortune» et des menées d'autrui, ils sont souvent eux-mêmes les premiers responsables?

Responsables, parce qu'imprudents, imprévoyants, mais aussi - ce qui n'est du reste pas étranger à leur imprévision - parce que mus par leurs désirs, leurs affects, leurs passions. Celles-ci jouent sur la vaste scène du monde, comme pour la mécanique narrative elle-même (aussi bien du récit-cadre que des nouvelles), un rôle moteur. Le mot lui-même de «passion», toutefois, n'est pas d'un emploi très fréquent dans le Décaméron ${ }^{7}$. Une douzaine d'occurrences en tout et pour tout ${ }^{8}$, dont

4 Telle est la grande leçon du métadiscours qui encadre la première nouvelle (fait exceptionnel, cette nouvelle se conclut par un long commentaire du narrateur qui reprend et précise le propos introductif) ; cf. plus particulièrement les paragraphes 3-6 et 89.

${ }^{5}$ Selon la célèbre formule de F. De Sanctis dans sa Storia della letteratura italiana (18701871), pour laquelle je renvoie à l'éd. a cura di L. Russo, Milano, Feltrinelli, 1960², I, p. 335.

${ }^{6}$ Cent nouvelles, plus une, «inachevée»: celle que propose l'auteur lui-même dans l'introduction à la quatrième journée. Nous y reviendrons.

7 La remarque vaut pour l'ensemble de l'œuvre en langue vulgaire, soumise à un dépouillement conduit à partir de la Letteratura Italiana Zanichelli in CD-Rom (LIZ 3.0), a cura di P. Stoppelli ed E. Picchi, 2, Da Petrarca all'Umanesimo, Bologna, Zanichelli, 1998. C'est ce même outil qui sera utilisé par la suite pour les données quantitatives sur lesquelles s'appuiera l'analyse. Une étude systématique de la question devrait évidemment inclure l'œuvre latin, notamment le De mulieribus claris et le De casibus virorum illustrium. Une indication, tout de même: dans le sillage en particulier de Pétrarque (cf., de ce dernier, l'aboutissement d'une longue réflexion sur l'interprétation allégorique de Virgile dans la Senilis, IV 5, notamment les § 13-14), la défense de la poésie du livre XIV des Genealogie deorum gentilium entend justifier l'invention poétique de l'Énéide en ces termes (XIII, 16) : quod sub velamento latet poetico, intendit Virgilius per totum opus ostendere quibus passionibus humana fragilitas infestetur, et quibus viribus a constanti viro superentur. Et cum iam non nullas ostendisset, volens demonstrare quibus ex causis ab appetitu concupiscibili in lasciviam rapiamur, introducit Didonem. Un autre passage intéressant des mêmes Genealogie se trouve au livre VII (IX, 5), lorsque Boccace interprète, dit-il, les formes changeantes de la figure de Protée comme les passions par lesquelles les hommes sont tourmentés. Je cite d'après Tutte le opere di Giovanni Boccaccio, a cura di V. Branca, vol. VII-VIII, Genealogie deorum gentilium, a cura di V. Zaccaria, Milano, Mondadori, 1998, respectivement t. 1, p. $730-732$ et t. 2 , p. 1448. 
cinq relatives à la Passion de notre Seigneur. Pour ce qui est des autres, on peut les subdiviser comme suit : cinq concernent l'amour ; une, plus «technique », définit les impressions reçues en rêve pendant le sommeil ; la dernière est employée dans le sens premier de «souffrance physique et morale». Il n'est guère étonnant que l'amour occupe une telle place dans le Décaméron: non seulement deux journées du recueil sont consacrées aux amours et aux amants, mais à un titre ou à un autre, ce sont presque les trois quarts des nouvelles qui le mettent en jeu'. Mon propos n'est pas de m'attarder ici sur cette question, mais une indication d'ensemble pourrait être utile pour la suite. Boccace en effet donne dans le Décaméron un congé souvent nostalgique, parfois cruel, à l'amour courtois ${ }^{10}$, y compris dans son avatar ultime, poétique, du Dolce Stil Novo. L'amour est pour lui d'abord la force naturelle la plus puissante dans l'ordre humain, à laquelle il est vain de s'opposer ${ }^{11}$, et qui va de la concupiscence charnelle la plus élémentaire (ce péché si naturel) ${ }^{12}$ à des formes inédites de sublimation ${ }^{13}$. Les deux extrêmes ne sont pas même contradictoires : voyez à ce propos, dans la nouvelle initiale de la quatrième journée qui réécrit le motif du "cœur mangé $»^{14}$, le morceau d'éloquence d'une exceptionnelle bravoure, digne du plus valeureux des maîtres de l'ars dictaminis, que la jeune veuve Ghismonda, contre l'usage convenable d'ordinaire à son $\operatorname{sex}^{15}$, prononce pour justifier auprès de son père se sentant bafoué, ses amours avec un jeune valet, noble

${ }^{8}$ I 10, 12 ; II conclusion, 5 ; III 4, 18 ; IV 2, 10 ; VII conclusion, 16 ; VIII 7, 129 ; X 6, 27 et $35 ;$ X 8, 25 et 26 ; conclusion de l'auteur, 24.

${ }^{9}$ La famille lexicale de l'amour y compte plus de huit cent trente occurrences.

${ }^{10} \mathrm{Cf}$. par exemple les deux nouvelles emblématiques (V 8, la nouvelle de Nastagio degli Onesti; V 9, celle de Federigo degli Alberighi) qui concluent la deuxième journée entièrement consacrée à l'amour (comme pour toutes les journées à thème imposé, la dixième et dernière nouvelle jouit en effet d'un statut particulier, libre qu'elle est de toute contrainte thématique).

${ }^{11}$ Telle est notamment la grande leçon de la nouvelle prétendument incomplète qui ouvre la quatrième journée, insérée dans l'introduction, due ici, non pas comme d'habitude au narrateur de premier niveau, mais à la voix de l'auteur lui-même, qui lui assigne une fonction proprement stratégique, tant sur le plan métanarratif que pour sa leçon morale.

${ }^{12}$ Pour cette formule, cf. III 8, 45. Beaucoup plus tard, lorsqu'à la fin de sa vie Boccace entreprendra d' «exposer » publiquement la Comédie de Dante, il évoquera à propos des trois fauves du chant I de l'Enfer qui symbolisent la luxure, l'orgueil et l'avarice, la «passion » de la «lascivité », la «concupiscence charnelle » qui séduit tout au long de la vie la «sensualité humaine » par ses «mortelles douceurs », et dont la panthère est l'allégorie (cf. G. Boccaccio, Esposizioni sopra la Comedia, a cura di G. Padoan, Milano, Mondadori, 1965, I (II), «Esposizione allegorica », § 99, 107).

${ }^{13} \mathrm{Cf}$. notamment les nouvelles 5,6 et 7 de la journée IV. Sans oublier bien sûr la dernière nouvelle du Décaméron (X 10), celle qui devait connaître la fortune que l'on sait, Griselda devenant pour la France, grâce notamment à la traduction latine de Pétrarque, la Grisélidis (ou Griseldis, ou Gliselidis) de tant et tant de réécritures, à commencer par celle de Christine de Pizan dans la Cité des dames (II, L).

${ }^{14}$ L'autre version du «cœur mangé », plus adhérente à la tradition médiévale, est celle de la nouvelle IV 9, inspirée de l'histoire du troubadour Guilhem de Cabestanh.

${ }^{15}$ Cf. I 10, 4 ; VI 1, 3. 
non pas par la naissance, mais par sa vertu et ses manières. C'est un éloge vibrant de la chair conjuguée à la faculté de juger, un hymne à l'alliance de l'invincible « désir concupiscent» (qui n'a rien à voir, dit-elle, avec la «fragilité féminine») et des sentiments les plus élevés, fondés, sous le signe de la sagesse, sur l'élection de la $\operatorname{vertu}^{16}$. La passion, en l'occurrence amoureuse, peut être, on le voit, exaltée.

Mais parce qu'il ne saurait être question de passer en revue ici, pas plus que les manifestations de l'amour, l'ensemble des passions, envie, colère, haine, etc., et encore moins d'en dresser la systémique ou la logique, c'est à l'une d'entre elles que je voudrais m'attacher en particulier. Plus précisément, comme nous allons le voir, à l'une des passions «louables» de la liste que Dante avait fournie quatre décennies plus tôt dans le Convivio. C'est en effet un bref détour par ce dernier que je propose d'effectuer, afin de mieux saisir ce que pouvait être, sinon la pensée de Boccace en la matière, du moins l'horizon sur lequel se découpe pour lui la phénoménologie des passions. Ce n'est pas qu'en matière de passions, Dante fût la seule source de Boccace: celui-ci avait en effet recopié à la fin des années quarante le texte de l'Éthique à Nicomaque avec le commentaire de Thomas d'Aquin ${ }^{17}$. Mais la lecture de Dante avait nourri dès le tout début ses années d'apprentissage, Dante dont, disait-il, il tenait intégralement ce qu'il pouvait savoir du bien et qui lui était certainement le plus présent à l'esprit : avant tout l'auteur de la Comédie, certes, mais aussi celui du Convivio, le traité inachevé où est esquissée, plus particulièrement aux livres III et IV, une théorie des passions ${ }^{18}$.

Lecteur d'Aristote (l'Éthique à Nicomaque et la Rhétorique, essentiellement) et de Thomas d'Aquin (commentateur de l'Éthique), Dante considère d'abord l'altération qu'est la passion, enracinée dans la «vie sensitive» comme une «tache

\footnotetext{
${ }^{16}$ Le discours de Ghismonda occupe les paragraphes 31-45 de la nouvelle IV 1.

${ }^{17}$ Cf. Anna Maria Cesari, «L'Etica di Aristotele del Codice Ambrosiano A 204 inf. : un autografo del Boccaccio », Archivio storico Lombardo, XCIII-XCIV (1966-1967 [1968]), p. 69-100. Seul d'ailleurs le commentaire de saint Thomas serait autographe: cf. Ginetta Auzzas, «I codici autografi. Elenco e bibliografia », Studi sul Boccaccio, VII (1973), p. 1-20. Sur le substrat du Décaméron en matière de philosophie morale, cf. Francesco Bausi, Gli spiriti magni. Filigrane aristoteliche e tomistiche nella decima giornata del Decameron, Studi sul Boccaccio, XXVII, 1999, p. 205-253 ; Lucia Battaglia Ricci, «Decameron: interferenze di modelli », Autori e lettori di Boccaccio, Atti del Convegno internazionel di Certaldo (20-22 settembre 2001), a cura di M. Picone, Firenze, Cesati, 2002, p. 179-194. Pour une discussion d'ensemble sur la morale du Décaméron et l'«épicurisme» de Boccace, cf. Marco Veglia, «La vita lieta ». Una lettura del Decameron, Ravenna, Longo, 2000.

${ }^{18}$ Amorosa visione, V, 84-88 et VI, 2-3 («-Costui è Dante Alighier fiorentino, / il qual con eccellente stil vi scrisse / il sommo ben, le pene e la gran morte : / gloria fu delle Muse mentre visse $[\ldots]$ » « $\mathrm{Al}$ suon di quella voce graziosa / che nominò il maestro dal qual io/ tengo ogni ben, se nullo in me sen posa [...]»). Rappelons que Boccace fut le premier biographe de Dante, avec les trois rédactions du Trattatello in laude di Dante (cf. Tutte le opere, op. cit., vol. III : les différentes rédactions du Trattatello, a cura di P. G. Ricci, se trouvent aux p. 437538 ; dans la première, la plus longue, le Convivio est présenté à la p. 488). Cf. aussi les échanges avec Pétrarque au sujet de Dante et, en particulier, la Fam. XXI 15 adressée par Pétrarque à Boccace.
} 
de l'âme», comme ce qui égare le jugement, cause de ce fait de mauvaise réputation. L'amour, évidemment, en tant que désir de l'âme «passionnée» pour une créature sensible, occupe dans ce contexte une place éminente ${ }^{19}$. Refusant néanmoins la rigueur stoïcienne, parce qu'elle est contraire à la nature et, partant, vouée à l'échec, il considère, dans le sillage d'Aristote et, dit-il, d'Augustin, que les passions, notamment les «innées $»^{20}$, font partie de notre être, puisque celui-ci est constitué de l'union de l'âme avec un corps sensible. Dès lors, la juste voie consiste à les réfréner, à les dominer par l'habitus de la «rectitude» et de la «vertu» (mélange, cette dernière, de noblesse et de passion, où la première domine la seconde) : c'est ainsi que l'on évite de tomber dans l'habitus contraire du vice et de commettre le péché ${ }^{21}$. Tâche difficile, à remettre sans cesse sur le métier, a fortiori lorsque l'«agent» est plus proche du «patient» (Dante recourt ici au sens philosophique technique de la passion comme corrélat d'une action) : l'âme est alors plus «passionnée», plus tributaire de sa partie concupiscente ${ }^{22}$.

Certaines nouvelles du Décaméron paraissent illustrer parfaitement une telle conception. Il est vrai qu'on les trouve plutôt dans la dixième et dernière journée, celle à laquelle son thème imposé, libéralité et magnificence (ou magnanimité), confère un statut spécial, en quelque sorte de couronnement idéal de l'œuvre sous le signe de la vertu ${ }^{23}$. Un exemple suffira : celui de la nouvelle X 6, dans laquelle le roi

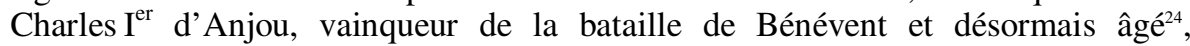
s'éprend «follement» d'une jouvencelle, fille d'un chevalier lui ayant offert l'hospitalité. Cette violente et secrète «affection» (un peu plus loin appelée «passion $»)^{25}$ amène le fidèle et proche compagnon à qui il s'ouvre de ses intentions

\footnotetext{
${ }^{19}$ Dante, Convivio, I II, 16 ; I IV, 10 ; III X, 1-2. Le désir et la haine, comme l'amour, sont des passions : cf. IV I, 2. Je renvoie à l'édition suivante, pour la richesse de son commentaire, en particulier en ce qui concerne les sources : Dante Alighieri, Opere minori, t. I, Parte II, a cura di C. Vasoli e di D. De Robertis, Milano-Napoli, Ricciardi, 1988 (ce fort volume ne comprend que le Convivio).

${ }^{20}$ Dante fait la distinction entre les passions «connaturali» et les «consuetudinarie»: cf. III VIII, 17-18. Dans ce passage, le mot «passion» est utilisé comme synonyme de « vice ».

${ }^{21}$ Cf. IV VI, 9 (pour les Stoïciens); IV XX, 2 (pour Aristote et Augustin) ; IV XXI, 14.

${ }^{22}$ Cf. III X, 2.

${ }^{23}$ Cf. le «titre» de la journée («nella quale [...] si ragiona di chi liberalmente o vero magnificamente alcuna cosa operasse intorno a' fatti d'amore o d'altra cosa »); la majorité des occurrences des deux familles lexicales ( «libéralité » et «magnificence ») se trouve dans cette dernière journée. Dans le Convivio IV XVII, 5, libéralité, magnificence et magnanimité (dans cet ordre) sont rappelées comme faisant partie des onze vertus de l'Éthique aristotélicienne, où elles figurent dans le même ordre. Mais, symptomatiquement sans doute, «magnanimité », mot clé chez Dante, n'est que très peu utilisé par Boccace (entre «magnanimo » et «magnanimità », quatre occurrences, dont trois dans la nouvelle VIII 7 ; une seule - «magnanimo »- dans la X 6).

${ }^{24}$ C'est ce que nous dit Boccace dès la «rubrique » initiale ; le roi Charles avait en réalité à peine quarante ans en 1266, année de la victoire sur Manfred,

${ }^{25}$ Cf. X 6, 24 et 27 . Le terme, ordinairement plus neutre (mais il renvoie parfois aux appétits naturels), est déjà utilisé dans ce sens de «passion amoureuse » dans la nouvelle II 8, 7 .
} 
à en appeler à sa raison. Le discours de celui-ci, rhétoriquement très élaboré, prend fin par l'exhortation suivante: «Je vous rappelle, sire, que ce vous est une grande gloire d'avoir vaincu Manfred, mais qu'il est beaucoup plus glorieux de se vaincre soi-même. Ainsi, vous qui avez à gouverner les autres, sachez d'abord vous vaincre et réfréner votre appétit $[\ldots] \gg$ Ce que mit à exécution le roi au prix d'un effort et d'une souffrance «inestimables»: il marie la jeune fille à un riche baron en la dotant «magnifiquement »; il sera désormais un homme libéré des chaînes d'une telle passion, et ce, pour le reste de ses jours, grâce toutefois à une lutte continuelle et à la macération de son farouche et inextinguible appétit.

Même s'il est possible de la dominer, la passion, pour Dante, reste foncièrement dangereuse pour l'âme : elle éloigne de la raison et, partant, des fins de la «bonne nature». Cependant, à diverses reprises, il envisage aussi de bonnes passions. Voici la liste des six passions spécifiques de l'âme humaine, fruit de la sélection qu'il opère dans le livre II de la Rhétorique aristotélicienne (qui, rappelons-le, en comprend neuf) : faveur, émulation, pitié, envie, amour, honte ${ }^{26}$.

\footnotetext{
${ }^{26}$ Cf. III VIII, 10 : «grazia, zelo, misericordia, invidia, amore e vergogna ». Pour l'équivalent français des ces termes, sauf pour l'amour (celui-ci constitue un cas à part, qui nécessiterait à lui seul de longs développements), j'utilise, par commodité et parce qu'il ne s'agit pas ici d'entrer dans les détails, l'éd. suivante: Aristote, Rhétorique, introduction de M. Meyer, Traduction de Ch.-E. Ruelle, revue par P. Vanhemelryck, commentaires de B. Timmermans, Paris, LGF (Le Livre de Poche, «Classiques de la philosophie »), 1991 (dans l'éd. des Belles Lettres, Aristote, Rhétorique, texte établi et traduit par M. Dufour, Paris, 1938, II, la série donne ceci, selon l'ordre tel que remanié par Dante: obligeance, émulation, pitié, envie, amitié, honte). Mais, pour prendre l'exemple le plus flagrant (en dehors de l'amour, en lieu et place d'《amitié»), le recours à «pitié» (le grec éléos) pour traduire misericordia, pose problème, puisque Dante prend bien soin de distinguer la «pitié», «noble disposition de l'âme », de la «miséricorde », passion qui en est un effet spécial (cf. II X, 6). Chez Guillaume de Moerbeke (Dante est sans doute tributaire de la traduction latine fournie par ce dernier vers 1270 : cf. E. Berti, Enciclopedia dantesca, Roma, Istituto della Enciclopedia Italiana, 1984, IV, «Retorica », p. 892b-893b), la série donne ceci : «gratia», « zelus », « misericordia », «invidia», «amicitia» et «erubescentia» (cf. Aristoteles latinus XXXI 1-2: Rhetorica. translatio anonyma sive vetus et Translatio Guillelmi de Moerbeka, ed. B. Schneider, Leiden, E.J. Brill, 1978; on notera cependant - y compris comme pièce à verser au dossier des sources de Dante - que la Translatio anonyma traduit le grec aïschynè par verecundia, étymon de «vergogna »). Dante écarte donc de la liste aristotélicienne la colère (l'ira au demeurant est un vice, auquel s'oppose la vertu de mansuétude : cf. IV XVII, 5 -même si en III VIII, 17-18, où elle apparaît aussi, la frontière entre vice et passion est de nouveau loin d'être étanche), la crainte (il l'évoque comme telle - «temenza »- en II X, 5) et l'indignation (mais aussi la haine - c'est-à-dire l'envers de l'amour - qui est bien une passion, à l'instar du désir : cf. IV I, 2 ; elle peut toutefois être bonne passion, lorsqu'elle combat la «malizia » : IV I, 5) ; il ne dit pas explicitement en quoi elles sont propres à l'âme, mais on peut avancer l'explication suivante : c'est que les deux premières impliquent les opérations du corps (cf., encore pour la «complexion colérique », III VIII, 17) et que la dernière est, selon Aristote (et ses traducteurs), l'opposé de la «pitié »; quant à l'amour dont il est question ici, ce n'est pas
} 
Celles-ci, si l'on excepte l'envie, qui est aussi un vice capital, ressortissent à ce que Dante définit ailleurs comme passions «louables». L'amour et la «misericordia », en effet, dérivent de la pitié (cf. les précisions terminologiques de la note 26), «noble disposition de l'âme, prête à recevoir amour, miséricorde et les autres passions caritatives $»^{27}$. Quant à la honte, qui est aussi désignée comme "pudeur $»^{28}$, elle fait partie, avec la «misericordia » et beaucoup d'autres, des passions positives, précisément, qui resplendissent dans l'âme noble ${ }^{29}$. Bonne passion (et non pas vertu $)^{30}$, elle est crainte du déshonneur, en particulier chez les jeunes gens et les femmes $^{31}$. Elle est même tout à fait nécessaire chez les «adolescents »: elle se subdivise, pour eux tout spécialement, en trois passions distinctes qui sont au fondement de la vie bonne, à savoir l'admiration («stupore»), la pudeur («pudore») et le sentiment de honte («verecundia»)- ces deux derniers extérieurement reconnaissables au blêmissement et au rougissement ${ }^{32}$.

celui qui procède de la concupiscence, mais d'abord l'amour de la sagesse, passion spécifique de l'espèce humaine (III XI, 7).

${ }^{27}$ Cf. II X, 6.

${ }^{28}$ Cf. III VIII, 10 : c'est parce que la «vergogna », comme les autres passions de l'âme, se manifeste dans le regard, qu'CEdipe s'est privé de la vue et «con etterna notte solvette lo suo dannato pudore. »

${ }^{29}$ Cf. IV XIX, 5.

${ }^{30}$ On reconnaît là la thèse développée dans le chap. 15 du livre IV de l'Éthique à Nicomaque (voici le début du chapitre dans la version de J. Tricot, Aristote Éthique à Nicomaque, traduction et notes par J. Tricot, Vrin, 1959: «En ce qui concerne la modestie il ne convient pas d'en parler comme d'une vertu, car elle ressemble plutôt à une affection qu'à une disposition Quoiqu'il en soit, on la définit comme une sorte de crainte de donner une mauvaise opinion de soi et elle produit des effets analogues à ceux que provoque la crainte du danger : on rougit, en effet, quand on a honte [le verbe est celui qui correspond au substantif aïschynè rencontré à la note 26], et on pâlit quand on craint pour sa vie. Dans un cas comme dans l'autre, il semble donc bien qu'il s'agisse là de quelque chose de corporel en un sens ce qui, on l'admet communément, est plutôt le fait d'une affection que d'une disposition. L'affection en question ne convient pas à tout âge, mais seulement à la jeunesse. Nous pensons que les jeunes gens ont le devoir d'être modestes, parce que, vivant sous l'empire de la passion, ils commettent beaucoup d'erreurs, dont la modestie peut les préserver; et nous louons les jeunes gens quand ils sont modestes». On relèvera que «modestie » (aïdôs) est rendu par «pudeur»dans Aristote, Éthique de Nicomaque, traduction, préface et notes par J. Voilquin, Paris, Garnier, 1965).

${ }^{31}$ Cf. IV XIX, 8.

${ }^{32} \mathrm{Cf}$. IV XXV, 3-10 : «[...] lo stupore è uno stordimento d'animo, per grandi e maravigliose cose vedere o udire o per alcuno modo sentire : che, in quanto paiono grandi, fanno reverente a sé quelli che le sente ; in quanto paiono mirabili, fanno voglioso di sapere quelle »; «Lo pudore è uno ritraimento d'animo da laide cose [au premier chef, l'amour vénérien], con paura di cadere in quelle»- et quelques lignes plus loin: «Oh quanti falli rifrena esto pudore ! - ; «La verecundia è una paura di disonoranza per fallo commesso ; e di questa paura nasce un pentimento del fallo, lo quale ha in sé una amaritudine che è gastigamento à più non fallire. » Pour Dante, l'adolescence va jusqu'à vingt-cinq ans (IV XXIV, 4). 
Voyons alors ce qu'il en est chez Boccace. Mais pourquoi nous attacher plus particulièrement désormais à la «vergogna»? Outre le fait qu'il vient moins spontanément à l'esprit de la considérer comme une passion (ce serait aussi d'ailleurs le cas de la faveur ou de l'émulation), il y a d'abord une raison statistique. La seconde raison se déduira - j'espère - de l'analyse elle-même.

En dehors de l'amour, c'est en effet l'affect qui, mauvaises passions comprises, revient le plus souvent, avec au total plus de cent quarante occurrences de la famille lexicale concernée - auxquelles s'ajoutent six emplois de «onta», deux fois d'ailleurs en binôme avec « vergogna ». Loin devant par exemple la miséricorde ou l'envie (autour d'une vingtaine d'occurrences chacune) ) $^{33}$. Elle concerne ensuite l'ensemble des journées du recueil - même si la répartition est quelque peu inégale ${ }^{34}$. Quelles sont alors les lignes de force qui se dégagent d'un examen un tant soit peu systématique des usages en contexte des mots de la famille en question?

Rappelons-nous ce que disait Dante de ceux chez qui elle est particulièrement souhaitable. Les femmes occupent bien une place légèrement majoritaire (une petite cinquantaine $^{35}$ dans la distribution d'ensemble que réserve à cette passion le Décaméron. En revanche, parmi les protagonistes de sexe masculin, les jeunes gens doivent se contenter de la portion congrue : ils sont moins d'une dizaine à éprouver un tel affect $^{36}$. Mais l'essentiel est ailleurs. La «vergogna» n'a que rarement la même signification selon que l'on est homme ou femme. Nous le verrons bientôt.

Au préalable, une dernière précision d'ordre général. Nous serons amenés en effet à écarter du champ de la réflexion la première acception (selon la décomposition analytique du concept proposée par Dante), qui est à vrai dire une sorte d'hapax ${ }^{37}$ : non seulement l'admiration (le «stupore» du Convivio) n'est pas un affect courant dans le monde du Décaméron, mais elle se limite pour l'essentiel à ce

\footnotetext{
${ }^{33}$ En marge de la série des passions de l'âme selon Dante, «pietà » (avec ses dérivés, une soixantaine d'occurrences), «compassione» (quarante-cinq) et «misericordia » (vingt-cinq) mériteraient une enquête spécifique. «Ira» et sa famille reviennent à une soixantaine de reprises.

${ }^{34}$ Les deux journées qui offrent le plus d'occurrences sont la première et la dernière (vingtquatre chacune), suivies de la cinquième (dix-huit), de la deuxième (dix-sept) et de la troisième (seize). Celle où la «vergogna » apparaît le moins souvent (quatre fois seulement) est la sixième (mais c'est aussi celle dont les nouvelles sont de très loin les plus courtes). Pour esquisser une corrélation possible avec les thèmes des journées : la première est celle qui trace les grandes orientations du recueil ; la dernière est celle où se manifestent les plus hautes vertus; quant à la cinquième, celle des amours qui finissent bien, il se pourrait que la « vergogna » ait son rôle à jouer dans les issues heureuses.

${ }^{35}$ Ce chiffre n'est pas à rapporter au total des occurrences, puisque celles-ci sont susceptibles de concerner plusieurs fois un même personnage.

${ }^{36}$ Quelques rares énoncés au statut métadiégétique, enfin, ont, en ouverture ou en conclusion des nouvelles, une portée parémiologique, applicable au genre humain tout entier, sur le fond de laquelle s'inscrivent donc les situations particulières évoquées dans les récits: ainsi de III 2,$3 ; \mathrm{X} 8,112$.

${ }^{37}$ Le Grande Dizionario della Lingua Italiana, Fondato da S. Battaglia, dir. Scientifico G. Barberi Squarotti, Torino, UTET, 1961-2002, ad vocem «vergogna », n'enregistre même pas cette acception.
} 
qui, par son caractère apparemment surnaturel, frappe l'imagination, notamment des foules crédules $^{38}$. Il est vrai, d'autre part, que l'émulation caractérise plusieurs nouvelles de la première journée et, surtout, de la dixième et dernière, mais elle ne concerne que très peu le «désir d'apprendre » propre aux «adolescents » évoqué par Dante $^{39}$. Il n'y a qu'une nouvelle (X3) qui pourrait faire exception: nous l'évoquerons un peu plus tard. C'est donc pour le moment entre honte et pudeur, voire, au sens actif, «offense» destinée à humilier celui qui en est la victime, que nous allons voir se déployer le spectre de cette passion.

Le sentiment de «vergogna » qu'éprouvent les hommes est principalement la honte. Une honte avant tout sociale, dans laquelle évidemment les jeux de l'amour, dans toute l'étendue du concept selon le Décaméron, occupent une place prépondérante: maris trompés, qui souvent cherchent précisément à éviter le déshonneur ou à le venger ${ }^{40}$; pères ou frères ${ }^{41}$ dont les filles ou les sœurs bafouent le renom familial en s'énamourant de jeunes gens de condition inférieure, s'exposant ainsi à des conséquences parfois extrêmes ${ }^{42}$. Lorsque les usages sociaux sont ainsi méprisés, voire bravés, la honte, ou la crainte de la honte ${ }^{43}$, sont, plus particulièrement lorsque nous avons affaire aux personnages masculins, un ressort de la narration, elles déterminent leurs décisions, les actions qu'ils entreprennent généralement peu inspirées, car irréfléchies, mues justement par une passion incontrôlée. La honte est aussi peur d'une dégradation, non provoquée par autrui, de l'image de soi, que l'on voudrait préserver aux yeux du monde ${ }^{44}$. Les mots peuvent être dans ce cas des armes redoutablement efficaces (c'est le thème de la sixième

\footnotetext{
${ }^{38}$ Voir par exemple III 8, 73 ou VI 10, 53. Le cas de Cimone (V 1, 8), seul protagoniste du Décaméron à tomber en admiration (qui est en réalité hébétude) devant la beauté féminine ressortit davantage à la logique de l'état de stupeur caractéristique de l'amor hereos. Quant à «stupore » et les adjectifs dérivés, ils sont quasiment absents de l'œuvre.

${ }^{39} \mathrm{Ce}$ qui pourrait susciter un tel affect, magnificence, magnanimité et libéralité, voit les mots qui le disent très majoritairement concentrés dans la dixième et dernière journée («splendore » et dérivés sont en revanche d'un emploi très rare). Mais pour exemplaires que soient ces nouvelles, elles ne sont guère reconductibles à la logique de la «vergogna »émulation, exception faite (mais il ne s'agit pas de jeunes hommes) des nouvelles 5 ( $\$ 25$ ) et 9 (§58).

${ }^{40}$ Ainsi de II 9, 62 (le marchand Bernabò da Genova est «vinto dall'ira della perdita de' [suoi] denari e dall'onta della vergogna che [gli] parea avere ricevuta dalla [sua] donna »); III 2,19 ; etc.

${ }^{41}$ On sait depuis le «Proemio », 10, que ceux-ci (comme d'ailleurs les mères - souvent plus compréhensives, cependant - et les maris) gardent les jeunes femmes jalousement enfermées dans leurs « camere », où elles se morfondent.

${ }^{42}$ IV 1, 43 ; IV 5, 7; V 4, 43 ; VIII 8, 9.

${ }^{43}$ III $2,19,30-31$.

${ }^{44}$ Le prévôt surpris dans les bras d'une servante monstrueusement laide, alors qu'il croyait être dans ceux d'une belle veuve (VIII 4, 34); Calandrino bafoué par ses amis (VIII 6, 48); Salabaetto, berné lui aussi, par une belle Sicilienne (VIII 10, 40).
} 
journée) ${ }^{45}$. La honte peut être enfin le fruit d'une perte «historique» de prestige social : en pareil cas, soit elle est un sentiment que le sujet de cet affect garde pour lui-même, soit, parce qu'elle met en jeu un motif noble, comme l'incapacité d'assumer les obligations d'un amour «courtois», elle pousse à inventer une solution inédite ${ }^{46}$.

Il arrive que ce sentiment éminemment «social»soit éprouvé par les femmes, pour des raisons plus ou moins honorables - à cette différence près que, dans leur cas, qu'elles soient victimes des coups d'une fortune contraire ou la cause même du danger encouru, le sort fâcheux qu'elles sont susceptibles de subir, autrement dit la «vergogna», est toujours directement liée aux actes qu'elles sont amenées à commettre elles-mêmes. Un tel sentiment, fondé sur la crainte de l'infamie potentielle, est donc susceptible de déterminer également les actions et les comportements féminins ${ }^{47}$. C'est ainsi que la protagoniste d'une nouvelle de beffa est mue par le désir d'épargner à son couple l'opprobre des Arétins; qu'une autre invente un stratagème pour se prémunir des conséquences possibles du mauvais pas dans lequel elle s'est trouvée impliquée malgré elle ; que la femme défigurée parce qu'elle n'a pas écouté les conseils de son mari ne veut plus se faire voir en public ${ }^{48}$. En ce sens, d'ailleurs, «vergogna » peut prendre un sens transitif : pour se venger, l'on cherchera a «far vergogna » à celui dont on a subi l'offense (elle-même au demeurant définie parfois comme «vergogna» ${ }^{49}$. Objectivée, elle revient enfin régulièrement dans la bouche des narrateurs pour stigmatiser actions et comportements individuels dignes de blâme, ou pour brocarder les mœurs contemporaines ${ }^{50}$.

Pour les deux sexes aussi, la «vergogna » peut être remords - verecundia au sens de Dante. Cela se produit lorsqu'il y a infraction aux règles de la courtoisie: ainsi des railleries injustes d'une femme (et de ses amies) lancées à l'encontre d'un vieil homme quant à lui parfaitement «courtois $»^{51}$; de l'absence de libéralité ou de l'avarice d'Ermino de' Grimaldi' ${ }^{52}$. Les fautes commises à l'encontre des codes sociaux en vigueur se colorent alors de connotations morales : ce qui advient quand on fait reproche à autrui de ce dont on s'est rendu coupable soi-même ${ }^{53}$; si l'on se

\footnotetext{
${ }^{45}$ Ainsi du prélat et de son compagnon qui ont offensé l'honnêteté d'une jeune Florentine, et essuient une réplique cinglante, «mordante » (VI 3, 11).

${ }^{46}$ Pour le premier cas, cf. IX 4, 24 ; pour le second, la nouvelle déjà citée IV 9.

${ }^{47}$ II 6, 8 ; IV 10, 17 ; V 2, 19 ; V 4, $11 ;$ X 5, 13.

${ }^{48}$ VII 4,13 et 16 ; IX 6, 27 ; IX 7, 14.

${ }^{49}$ Ainsi (pour «far vergogna ») en I 4, 14 ; I 9, 5 ; II 8, 95 ; III 6, 20-21 ; IV 1, 35 ; V 3, 25 (il s'agit ici d'agression physique sur une femme) ; VII 4, 16, 24 ; VII 7, 43 ; VIII 6, 43 (et la variante «svergognare » en III 6, 34). Dans le sens d'offense (susceptible d'engendrer la honte), cf. I 8, 9; I 9, 5 (couplé à «onta »); II 6, 48; II 9, 62 («onta della vergogna »); III 2, 3 ; III 3, 45 ; IV 1, 19, 22 ; VIII 7, 98 ; VII 9, 77.

${ }^{50}$ I 8, 7, 10 ; I 10, 4, 19; VI 1, 3. Ce sens objectif peut aussi être utilisé hypocritement par un personnage à l'encontre d'un tiers absent pour faire croire à sa propre vertu (V 10, 44).

${ }^{51} \mathrm{I} 10,19$

${ }^{52}$ I 7, 25 ; I 8, 17.

${ }^{53} \mathrm{I} 4,22$.
} 
repent d'avoir injustement maltraité quelqu'un ${ }^{54}$, en se méprenant sur son compte ou en trahissant l'amitié ${ }^{55}$, ou d'avoir prononcé indûment des mots injurieux (et qui peuvent avoir appelé une réplique à son tour blessante $)^{56}$. Tel est le cas encore avec l'injustice cruelle commise par Bernabò à l'endroit de sa femme ${ }^{57}$; avec la tromperie croisée d'une épouse volage, de son mari et de son amant ${ }^{58}$. Il arrive même que telle narratrice porte sur ses épaules la faute collective commise par ses semblables ${ }^{59}$. Mais on voit alors qu'il y a là changement de registre: de sociale qu'elle était, fondée sur la peur du qu'en dira-t-on, la honte relève maintenant d'un mouvement intérieur ; elle est prise de conscience, souvent grâce à l'action ou aux dires d'autrui, d'une infraction coupable à la morale, qui remet en cause l'image de soi de qui l'a commise; et elle appelle réparation ${ }^{60}$. Telle était la grande leçon de la nouvelle évoquée plus haut, mettant en scène le roi Charles d'Anjou, que retient la honte de sa «folle pensée», de ce qu'il a seulement désiré ${ }^{61}$. Alors que la «vergogna» envisagée précédemment était le plus fréquemment source de disgrâces, l'affect qui procède du dialogue intime avec soi-même, nourri des «lois divines ou humaines ${ }^{62}$, porte à la restauration de l'harmonie, à la disparition de ce qui a troublé l'ordre de la vie associée, des rapports humains. Selon la théorie de matrice aristotélicienne évoquée plus haut, s'il avait été parfaitement vertueux, le roi, vu son âge, n'aurait jamais dû se retrouver dans une telle situation, source de «vergogna ». Mais l'affect éprouvé est propédeutique et c'est sur un nouvel habitus, celui de la conscience renouvelée de l'affect premier, que se conclut la nouvelle: la «vergogna», de passion qu'elle était, se transforme en quasi vertu et Boccace retrouve - sans même le savoir, sans doute - la «ligne romaine» (celle de la «vergogna »-vertu, précisément) dont parle Paolo Cherchi à propos de Pétrarque ${ }^{63}$. Il est un autre cas remarquable, qui mérite qu'on s'y arrête un instant.

La nouvelle X 3 , au chronotope incertain, située dans un Orient à demi fabuleux (le Catay, jadis), met en scène un jeune homme riche (Mitridanes), envieux de la réputation de grande vertu d'un vieillard (Natan) dont la libéralité et la munificence sont inégalables. Cette passion (l'envie), qui aurait pu être motif d'émulation, se transforme vite en fureur et il décide d'éliminer de ses mains ce rival lorsqu'il s'aperçoit que sa propre «fama» n'atteindra jamais les mêmes sommets. Le vieil homme, qui se fait passer pour un de ses propres domestiques, et pour

\footnotetext{
${ }^{54} \mathrm{X} 5,20$.

${ }^{55}$ II 6, 74 ; III 7, 99 ; X 3, 32, 39.

${ }^{56}$ II 8,94 ; VI 9, 15.

${ }^{57}$ II $9,69$.

${ }^{58}$ VIII 8, 32.

${ }^{59} \mathrm{I} 10,4$.

${ }^{60}$ Dans le registre des ébats amoureux entre jeunes gens - amour qui sera in fine légitimé par la conclusion de la nouvelle -, le narrateur de la V 4 évoque « la vergogna del fallo commesso e la voglia de lo emendare » ressenties pas la jeune fille au moment où elle est surprise par son père dans une situation bien embarrassante $(\$ 45)$.

${ }^{61} \mathrm{X} 6,1$.

${ }^{62}$ Ces lois que dissout à Florence la peste : cf. I Introduzione, 23.

${ }^{63}$ Cf. Paolo Cherchi, Verso la chiusura. Saggio sul «Canzoniere » di Petrarca, Bologna, Il Mulino, 2008, chap. I, « Di me medesmo meco mi vergogno», p. 19-48.
} 
lequel, en raison de sa parfaite serviabilité, Mitridanes se met à éprouver une révérence filiale, se propose même d'aider dans son entreprise celui qui veut l'assassiner. Mais dès que le jeune homme, découvrant l'identité véritable de son interlocuteur, se rend compte de ce que la libéralité de Natan va, pour ne rien avoir à refuser jamais à une requête de faveur, jusqu'au sacrifice de sa propre vie, il est pris d'un accès de honte ( «vergogna » $)^{64}$ qui fait retomber aussitôt son ire furieuse et l'envie mauvaise par quoi étaient offusqués les « yeux de son intellect». Débarrassé de son «désir pervers», il accepte désormais que le vieil homme lui soit un père, perçu comme un modèle, et la nouvelle se termine par un échange amène, presque badin, où les deux personnages se retrouvent sur un même pied, faisant l'un l'autre assaut de courtoisie. Dans ce brusque éveil de la conscience, provoqué par l'extrême vertu d'autrui, entre donc quelque chose comme de l'admiration : l'«envie», en soi ambivalente $^{65}$, avait conduit d'abord Mitridanes sur la pente fatale d'un acte abominable. Grâce à la honte, née sur un terreau dont on déduit à présent qu'il était a priori favorable, mais masqué par de violentes passions concurrentes, elle nous porte à proximité de la bonne passion («stupore ») évoquée par Dante.

Si l'on examine à présent ce qui caractérise plus particulièrement la «vergogna» au féminin, on s'aperçoit qu'elle penche nettement du côté de la pudeur et de la retenue, du «frein » que la conscience oppose à la tentation de la mauvaise action ${ }^{66}$. La crainte du déshonneur, ou le sentiment de ce qui n'est pas bon à dire ${ }^{67}$, sont fondés ici sur un sentiment «naturel », qui ressortit à l'intériorité de la conscience comme théâtre d'un dialogue intime, nourri de la perception de la faute, commise ou à venir. S'y mêle le cas échéant le repentir pour certaines de s'être mises par imprudence dans une situation inextricable, qui oblige à choisir entre le manquement à la parole donnée et l'action coupable ${ }^{68}$. C'est une caractéristique des femmes «sages »- car il en est de folles aussi ${ }^{69}$. Toutes, y compris ces dernières, sont cependant soucieuses de préserver leur honneur et se prêtent alors à mille tours hypocrites $^{70}$; ou, quand bien même elles restent persuadées de leur bon droit (si par exemple on les a mariées à un vieil homme, qui ne rend pas justice aux nécessités de la nature chez une femme jeune, fraîche et de solide constitution), elles ne peuvent s'empêcher d'éprouver une gêne: preuve que la conscience de la faute est intériorisée ${ }^{71}$. La honte d'avoir été surprise en mauvaise posture se double d'un sentiment de culpabilité - ce qui n'empêche pas d'essayer de sauver la mise (sociale et amoureuse $)^{72}$.

${ }^{64} \mathrm{Cf} . \S 27$.

${ }^{65}$ Voir en particulier ce que dit Natan au $§ 20$.

${ }^{66} \mathrm{Cf}$. la définition de Dante à la note 32 ci-dessus.

${ }^{67}$ Comme dévoiler les curieuses pratiques sexuelles d'un mari (IX 3, 22).

${ }^{68} \mathrm{X} 5,13$.

${ }^{69}$ II $9,18$.

${ }^{70}$ Seul ce qui est patent est motif de honte, suggère même un personnage, masculin, à vrai dire, qui médit des femmes : II 9, 19.

${ }^{71}$ II $9,19$.

${ }^{72} \mathrm{Cf}$. le cas de la nonne de IX 2, 14. 
Et c'est parce qu'elle est ivre que la Sarrasine Alatiel, qui prise le vin ${ }^{73}$ et qui de surcroît, passant ensuite de main en main, finit à chaque fois par y trouver son compte, oublie l' «honnêteté » et ses bonnes résolutions (ne connaître que le mari qui lui a été destiné), et se déshabille entièrement, sans aucune «retenue de pudeur $»^{74}$, devant le premier des amants choisis pour elle par la «fortune». L'héroïne de cette nouvelle, celle par excellence des continuels et stupéfiants revers de fortune (tel est en effet le thème de la deuxième journée), est victime de sa grande beauté et de l'appétit féroce et irrépressible des hommes - y compris les plus grands, de sang princier notamment, agités de passions violentes comme la colère aveugle, qu'ils ne cherchent pas même à contenir et qui les pousse aux meurtres les plus sauvages. Après cette première expérience, elle connaît huit (ou neuf) amants en quatre ans, et consomme l'acte charnel «dix mille fois $»^{75}$. Une intervention malicieuse du narrateur de premier niveau (celui du récit-cadre), au moment où il assure la transition au début de la nouvelle suivante, nous rappelle que ce qui concerne la chair a une force irrésistible : les soupirs poussés par les jeunes femmes de la «brigata» pourraient en effet bien être dus, autant qu'à la compassion éprouvée pour les «malheurs» d'Alatiel, au désir secret de «noces aussi fréquentes $»^{76}$. Expression muette de ce que la retenue empêche en tout état de cause d'articuler explicitement...

Car la pudeur, tout particulièrement celle que provoque la nudité, est innée chez les jeunes femmes - et il est en outre fort intéressant de constater que, dans le cas où un tel affect est partagé par un personnage de sexe masculin, il s'agit d'un homme jeune, d'un «adolescent $»^{77}$. C'est la raison pour laquelle aussi les mots trop crus suscitent l'embarras : la nécessité de désigner tout de même les choses conduit à l'invention de périphrases plus ou moins ingénieuses ${ }^{78}$. Une pudeur semblable retient de déclarer une flamme, ou en tout cas retarde l'échéance ${ }^{79}$. Cette forme de «vergogna » est elle aussi source de crainte, thématisée ainsi dès le «Proemio » et reprise dans la seule ballade chantée à l'intérieur d'une nouvelle ${ }^{80}$. Elle est

\footnotetext{
${ }^{73}$ L'amour du vin fait partie des «passioni consuetudinarie » de Dante (cf. Convivio, III VIII, 17). Plusieurs personnages boccaciens s'adonnent volontiers à la boisson, et généralement mal leur en prend (cf. les tours - «beffe »- que cela permet aux femmes de leur jouer : par exemple, VII 4, IX 6).

${ }^{74}$ «senza alcuno ritegno di vergogna » (II 7, 29).

${ }^{75}$ II $7,1,7,121$.

${ }^{76}$ II $8,2$.

${ }^{77}$ C'est ce qui arrive à une ingénue, convaincue qu'elle doit pour une bonne fin se soumettre aux volontés d'un abbé (III 8, 28); et cf. II 8, 51, 53 (Giachetto); V 3, 50 (Pietro Boccamazza) ; V 4, 44 ; V 5, 35 ; X 6, 17 ; X 10, 20 (il s'agit de Griselda) ; V 6, 29 (pour Gian di Procida et son amie, attachés nus au pilori).

${ }^{78} \mathrm{~V} 4,30$, VIII $5,3$.

${ }^{79}$ II 8, 8, 10 ; V 7, 6.

${ }^{80}$ Proemio, 10 et X 7, 20 (v. 13 de la ballade).
} 
également indice de modestie typiquement féminine ${ }^{81}$. Les manifestations de celle-ci comme de la pudeur s'accompagnent fréquemment de rougissements ${ }^{82}$.

Il y a là en réalité, à bien y regarder, quelque chose qui rentre dans un cadre programmatique, valable pour l'ensemble du Décaméron. L'absence de «vergogne», souvent soulignée, est source de débauche répréhensible, de dérèglements, tantôt mineurs, tantôt beaucoup plus graves ${ }^{83}$. Les «paliers» dont il était question en préambule sont à cet égard tout à fait significatifs. L'introduction à la première journée, pour évoquer la totale dissolution, provoquée par l'épidémie, de tous les liens sociaux, mentionne le cas de ces femmes qui, toutes nobles et belles qu'elles aient pu être, tombées brusquement malades, n'ont pas honte de mettre à découvert sous le regard des hommes leur intimité corporelle ${ }^{84}$. Et la première nouvelle du Décaméron est la démonstration éclatante de la ruine de tous les fondements du contrat social, à commencer par le pacte linguistique, à quoi aboutit une passion de ce type radicalement pervertie : Ciappelletto, le plus mauvais homme que la terre ait jamais porté, aurait eu honte de ne pas faire constamment le mal ${ }^{85}$; sur le point de mourir, dans sa parodie de confession, lieu de l'inversion ultime et blasphématoire de toutes les valeurs communément reçues, il ne cesse de feindre d'éprouver l'affect usuel ${ }^{86}$. Honte, donc, de ne pas être faux en toute chose, de ne pas commettre la fraude en toute circonstance (le pire des vices, source des pires péchés, rappelons-le, selon le Dante de la Comédie).

À l'opposé de cette nouvelle puissamment déstabilisante, l'institution des règles de fonctionnement de la «joyeuse compagnie» se fait, du côté féminin, sous le signe d'abord de la retenue, de la pudeur et de la crainte de l'opprobre ${ }^{87}$; puis,

${ }^{81}$ I Conclusione, 5 ; II 7, 93 (Alatiel elle-même !) ; VIII Conclusione 2 ; X 6, 13 ; et bien sûr, Griselda en X 10, 16.

${ }^{82}$ I Introduzione, 81 ; I Conclusione, 5 ; II 8, 10, 60 ; VI 8, 2 ; VIII Conclusione 2 ; IX 3, 22 ; X 7, 39 .

${ }^{83}$ Cf. le cas d'Alatiel tout juste évoqué ; c'est un groupe social bien particulier, celui des religieux, qui offre les exemples les plus frappants et nourrit de virulentes diatribes anticléricales : cf. I2, 19 (il s'agit des clercs de la Curie romaine, qui tous sans exception, passent leur temps à «peccare in lussuria, e non solo nella naturale ma ancora nella sogdomitica, senza freno alcuno di rimordimento o di vergogna ») ; III 7, 34 ; VII 3, 9-10.

${ }^{84}$ I Introduzione, 29.

${ }^{85}$ Le portrait de présentation de Ciappeletto, hyperbolique et insolitement long, occupe les § 10-15 de la nouvelle I 1 (la première phrase du premier de ces paragraphes précise d'emblée que «avea grandissima vergogna quando uno de' suoi strumenti [...] fosse altro che falso trovato »).

${ }^{86}$ Cf. ibid., $\S 37,67$.

${ }^{87}$ Neifile, la novice en amour et l'une des plus jeunes des sept femmes, «tutta nel viso per vergogna divenuta vermiglia» parce qu'aimée de l'un des trois jeunes gens venus les rejoindre, avait résisté dans un premier temps à l'idée exprimée par la plus âgée, la sage Pampinea, de partir ensemble se réfugier à la campagne, de peur que cela ne suscite «infamie et répréhension » (\$81-83). 
Pampinea, couronnée reine de la première journée ${ }^{88}$, esquisse les grands principes qui devront guider la vie du groupe : «dans l'ordre et le plaisir, et [...] sans honte aucune», le tout - avait-il été précisé avant le départ de Florence - «sans transgresser jamais en aucun acte les bornes de la raison ${ }^{89}$. À l'autre bout, ou presque, de la chaîne, je ne reviendrai pas sur la nouvelle finale (la victoire de Griselda est celle de la «patience» inspirée par la modestie), mais la X 8, parce qu'elle met en scène, dans sa première moitié, le combat intérieur qui déchire un jeune homme.

Dans une Antiquité gréco-latine magnifiée, le thème de l'amicitia est traité de la façon suivante: deux enfants, puis adolescents, sont élevés comme des frères. Mais lorsque le premier (l'Athénien Gisippo), est marié par sa famille à une jeune fille de merveilleuse beauté, le second (le Romain Tito) s'éprend follement d'elle. Plusieurs pages sont alors consacrées à la lutte entre, d'une part, la honte du second, incapable d'ouvrir les « yeux de l'intellect», d'obéir à la raison et de se vaincre luimême en réfrénant son appétit concupiscent, de résister à la tentation de l'autojustification au nom des lois de nature, et, d'autre part, ses devoirs de loyauté à l'égard de l'ami. Rougissant de honte, il finit, sur ses instances, par s'ouvrir à Gisippo de la «gravissime passion secrète $»^{90}$ qui le consume. L'amitié à toute épreuve qui unit ce dernier à Tito l'amènera alors, dans un élan de magnanimité mûrement pesée, à renoncer à l'épouse qui lui est destinée. Il y va de la santé, voire de la vie de son ami, d'autant plus sujet à la passion que son âme est plus noble. Après une ultime tentative de résistance opposée par la raison de Tito, la honte de l'un est vaincue par la libéralité de l'autre ${ }^{91}$.

Car c'est en vain que l'on s'oppose aux « raisons naturelles », dont les forces de l'amour, à côté de l'instinct de conservation, occupent le premier rang ${ }^{92}$. À l'intérieur de ce cadre, c'est une dialectique mouvante, à l'issue toujours incertaine, de l'appétit charnel et de l'honnêteté ${ }^{93}$, que met en jeu le Décaméron. En réalité, il

\footnotetext{
${ }^{88}$ N'oublions pas que les femmes, majoritaires, donneront sept « reines » sur les dix journées que compte le Décaméron.

${ }^{89}$ I Introduzione, respectivement aux $\S 98$ et 65 .

${ }^{90}$ Une retenue semblable, empêchant un jeune homme d'avouer sa flamme à son ami, parce qu'il s'est aperçu que celui-ci aime la même femme que lui (et l'ami est également honteux, car il s'agit d'un amour illicite), est - rapidement - pointée en VII 10, 12.

${ }^{91}$ Pour toutes les lignes qui précèdent, cf. X 8, 12-41. Un dernier accès de « vergogna » étreint Tito au $\S 48$, au moment où, grâce au subterfuge imaginé par Gisippo, il va rejoindre la belle dans son lit.

${ }_{92}$ Pour le syntagme «forza »- ou « forze »- «d'amor », on pourrait multiplier les exemples (II 8, 15; III 10, 3 ; IV Introduzione, 29; IV 1, 34 ; IV 7, 4; IV 8, 32 ; V 1, 2; V 6, 3 ; etc.). En ce qui concerne l'instinct vital, qui justifie le parti adopté par la compagnie des dix jeunes gens de quitter Florence, cf. I Introduzione, 53. Pour une utilisation tendancieuse des « raisons naturelles », cf. II 9, 13-15.

${ }^{93}$ On recense environ cent soixante-dix occurrences de la famille lexicale de l' «honnête » dans le Décaméron. Une analyse du concept mériterait d'être conduite, en particulier en relation avec la question de l' «honneur» (deux cent cinquante occurrences du terme et de ses
} 
est légitime de chasser la «vergogna» lorsque rien ne s'oppose à une union des cœurs et des corps ${ }^{94}$. À l'occasion de ses interventions d'auteur, Boccace précise bien qu'il refuse d'éprouver la moindre honte parce qu'il aime les femmes ${ }^{95}$. C'est dans la dernière acception ici examinée que la « vergogna » borde, en quelque sorte, le recueil: les narratrices, nous l'avons vu, en sont les garantes. Chez les femmes (nombre d'héroïnes aussi partagent ce trait), réside le plus haut degré de véritable sagesse, éminemment représenté par l'affect dont nous venons de nous occuper. Boccace donne ce faisant congé à toute une série de conventions sociales (essentiellement masculines) et de codes encore largement en vigueur dans la tradition littéraire. Pour pouvoir prétendre à une fonction régulatrice, tempérer comme il convient le nouveau jeu des rapports entre les humains, autrement complexe qu'auparavant, qui s'est instauré dans la société communale italienne et qui connaît l'une de ses crises les plus graves au milieu du Trecento ${ }^{96}$, il faut de bonnes passions (telles la pitié, la compassion, la miséricorde ${ }^{97}$, indissociables de l'«honnêteté » et de la «raison». Celle qui a fait l'objet de la présente enquête constitue un frein tout à fait efficace pour parer à l'entropie qui sans cesse menace les équilibres précaires. La «vergogna » féminine est exemplaire. Elle est l'un des signes les plus manifestes de l'action de la raison, instance toute pratique et discursive, dont l'opération consiste d'abord en un examen des situations empiriques et dont la finalité est d'empêcher la «folie», avec ses conséquences ravageuses. Elle est l'une des conditions premières de l'art de (bien) gouverner, tel qu'il est métaphorisé par le récit-cadre du Décaméron.

$\mathrm{Au}$ vieux mourant de la première nouvelle, s'opposent d'abord les jeunes femmes de la joyeuse compagnie, qui craignent d'éprouver, ne fût-ce qu'au simple récit d'aventures un tant soit peu osées, un plaisir illicite ${ }^{98}$. La licence revendiquée par Boccace de pouvoir tout représenter, incarnée tout particulièrement par Dioneo ${ }^{99}$, et qui est aussi licence de seconder les « raisons naturelles », lorsque ne s'y opposent pas les raisons impérieuses de l'honnêteté, est donc- littéralement et rigoureusement - encadrée. La honte, dès lors, pour le maître d'œuvre, ne réside pas dans l'objet de la représentation, mais dans la réception que l'on en fait. La «Conclusion de l'auteur» redouble par un métadiscours cette option audacieuse,

dérivés). Voir sur cette question Paolo Cherchi, L'onestade e l'onesto raccontare del Decameron, Fiesole, Cadmo, 2004.

${ }^{94}$ II 8, 53-54 ; IV 7, 9.

${ }^{95}$ IV Introduzione, 33.

96 Outre l'épisode ponctuel, mais oh combien traumatisant, de la peste noire, les «compagnies » florentines ont entamé dans la décennie concernée une phase de sérieux déclin.

${ }^{97}$ Voir la note 33 ci-dessus.

${ }^{98}$ Cf. I5, 1 (à la suite de la nouvelle précédente de Dioneo: cf. la note ci-dessous); $\mathrm{V}$ conclusione, 1 .

${ }^{99}$ Ce personnage malicieux, dont le nom dit bien sous quel signe il se place, jouit, rappelonsle, d'un privilège spécial, celui de conclure chaque journée sans avoir à en respecter le thème imposé. Dès la première nouvelle qu'il raconte (IV 4, quand la règle n'a pas encore été énoncée), et à l'exception de son sublime (ironique ?) rachat dans le dernier récit du recueil, il profite de cette liberté pour être particulièrement... licencieux. 
constitutive de l'idée même, tout à fait neuve, de produire un livre de nouvelles : Boccace nous dit qu'il n'aurait pas honte que ses nouvelles ne fussent pas toutes belles, parce qu'il s'est contenté de les recueillir et de les rassembler, et que seul Dieu est maître de perfection ${ }^{100}$. C'est le sentiment qui anime aussi l'une de ses narratrices, en préambule de la nouvelle II 4 (§4). On comprend mieux ainsi la stratégie oblique de Boccace, auteur du Décaméron. C'est à des femmes craintives et pudiques, tenant cachées dans leurs délicates poitrines les flammes amoureuses, qu'il adresse son livre ${ }^{101}$; toute nécessaire que soit à leur côté la présence des hommes, la majorité des narratrices sont des femmes et la première reine est une femme.

Si les passions chez Boccace aussi ont partie liée avec les vices et le péché ${ }^{102}$, il serait malaisé d'en formuler la théorie achevée, au sens rigoureusement philosophique et théologique du terme. C'est que cela ne l'intéresse guère. Ce qui ne veut pas dire qu'il n'y ait pas d'implications philosophiques (de philosophie pratique, non spéculative) dans la façon dont il les met en scène. Les passions sont ce qui détermine la marche du monde des hommes. Ce sont des passions qui, déclinées au féminin, permettent de la réguler, d'éviter la dissolution, comme elles régulent le «monde de l'œuvre». Alors que les autres passions (l'amour sensuel débridé, la haine, la colère, l'envie, etc., mais aussi la honte «sociale») sont des moteurs narratifs puissants, parce que porteurs de perturbations, la «vergogna», dans sa «bonne» acception, correspond au moment de la suspension, du retour réflexif, du jugement, elle permet d'éviter l'excès destructeur et rend possible le retour à des situations d'équilibre, d'inventer les bonnes solutions au fur à mesure des circonstances toujours inédites, au moins pour partie, auxquelles nous confronte le mouvement même de la vie, son flux inarrêtable.

Philippe Guérin

Université Rennes 2 - CELAM

\footnotetext{
${ }^{100}$ Conclusione dell' autore, 17.

${ }^{101}$ Proemio, 10.

${ }^{102}$ Cf. les Esposizioni, op. cit., I (II), «Esposizione allegorica », § 90 sq., dans le passage déjà cité sur les trois fauves du chant I de l'Enfer: luxure, orgueil et avarice, que ces animaux symbolisent, sont les vices dont Dante se savait le plus «passionato » (§91).
} 